\title{
Communication
}

\section{Strong Coupling between Plasmonic Surface Lattice Resonance and Photonic Microcavity Modes}

\author{
Yunjie Shi ${ }^{1,2}$, Wei Liu ${ }^{2,3}$, Shidi Liu ${ }^{2,4}\left(\mathbb{D}\right.$, Tianyu Yang ${ }^{2,5}$, Yuming Dong ${ }^{2,5}\left(\right.$, Degui Sun ${ }^{1, *}$ and Guangyuan Li $^{2,5}$ \\ 1 Schools of Science, Changchun University of Science and Technology, 7089 Weixing Road, \\ Changchun 130022, China; yj.shi@siat.ac.cn \\ 2 CAS Key Laboratory of Human-Machine Intelligence-Synergy Systems, Shenzhen Institute of Advanced \\ Technology, Chinese Academy of Sciences, Shenzhen 518055, China; liuwei@siat.ac.cn (W.L.); \\ liusd@siat.ac.cn (S.L.); ty.yang@siat.ac.cn (T.Y.); ym.dong@siat.ac.cn (Y.D.); gy.li@siat.ac.cn (G.L.) \\ 3 School of Physics and Electronic Engineering, Chongqing Normal University, Chongqing 401331, China \\ 4 Wuhan Research Institute of Posts and Telecommunications, Wuhan 430070, China \\ 5 Shenzhen College of Advanced Technology, University of Chinese Academy of Sciences, \\ Shenzhen 518055, China \\ * Correspondence: sundg@cust.edu.cn
}

Citation: Shi, Y.; Liu, W.; Liu, S.;

Yang, T.; Dong, Y.; Sun, D.; Li, G.

Strong Coupling between Plasmonic

Surface Lattice Resonance and

Photonic Microcavity Modes.

Photonics 2022, 9, 84 .

https://doi.org/10.3390/

photonics 9020084

Received: 30 December 2021

Accepted: 29 January 2022

Published: 1 February 2022

Publisher's Note: MDPI stays neutral with regard to jurisdictional claims in published maps and institutional affiliations.

Copyright: (c) 2022 by the authors Licensee MDPI, Basel, Switzerland. This article is an open access article distributed under the terms and conditions of the Creative Commons Attribution (CC BY) license (https:// creativecommons.org/licenses/by/ $4.0 /)$.

\begin{abstract}
We report the strong coupling between plasmonic surface lattice resonances (SLRs) and photonic Fabry-Pérot (F-P) resonances in a microcavity embedded with two-dimensional periodic array of metal-insulator-metal nanopillars. For such a plasmonic-photonic system, we show that the SLR can be strongly coupled to the F-P resonances of both the odd- and even orders, and that the splitting energy reaches as high as $153 \mathrm{meV}$ in the visible regime. Taking advantage of the strong coupling, the resulted high-energy upper polariton has similar characteristics as the plasmonic SLR, but the quality factor is almost twice of that of the SLR. We expect that this work will provide a new scheme for strong coupling between plasmonic and photonic modes, and will point to a new direction to improve the quality factor of SLRs.
\end{abstract}

Keywords: strong coupling; plasmonic-photonic coupling system; surface lattice resonance; Fabry-Pérot resonance

\section{Introduction}

Strong light-matter interactions involving confined electromagnetic fields in micro/nano-cavities and/or emitters, such as quantum dots [1], organic molecules [2] or transition metal dichalcogenides [3] are about the coherent exchange of energy between light and matter at resonance, leading to the formation of new hybrid states or particles called polaritons $[4,5]$. Among these, strong coupling between photonic cavities and plasmons have been of increasing interest [4,6-10] since the first demonstration between localized plasmons in nanowire pairs and photonic Fabry-Pérot (F-P) microcavity modes by Ameling and Giessen in 2010 [11]. By combining plasmonic nanostructures and photonic microcavities, it has been demonstrated that the sensing properties of localized plasmon sensors can be enhanced [12-14], that up to two-fold enhancement increase can be achieved compared to that without using the cavity [15], that large Rabi splitting can be obtained by embedding nanoparticle-WS $\mathrm{WS}_{2}$ heterostructure in an optical microcavity [16,17], and that both peak splitting and peak locking behaviors can be observed [18].

A key figure of merit for these strong coupling effects is the splitting energy. For example, Ameling and Giessen [11] showed that the splitting energy for the electric mode can be as large as $358 \mathrm{meV}$ in the near-infrared regime, corresponding to $36 \%$ of the resonant energy. Quite recently, Baranov et al. [19] further demonstrated ultrastrong coupling between nanoparticle plasmons and cavity photons at ambient conditions, and achieved giant Rabi splitting energy of $1 \mathrm{eV}$ and extremely large splitting/resonance energy ratio of $120 \%$. 
Besides the strong coupling between localized plasmons and photonic F-P cavity modes $[6,11,19]$, the strong coupling between other types of plasmons and other photonic modes has also been extensively investigated. Ameling et al. [20] demonstrated that both the localized and propagating surface plasmons can couple strongly with photonic microcavity modes, and the splitting energies were calculated to be $141 \mathrm{meV}$ and $224 \mathrm{meV}$, corresponding to $\sim 10 \%$ of the resonant energy in the near-infrared regime. Chen et al. [21] investigated the strong coupling between propagating surface plasmons and the F-P cavity and observed a large Rabi-analogue splitting energy of $148 \mathrm{meV}$ in the visible regime. Wang et al. [22] adopted another configuration and obtained $31.5 \mathrm{meV}$ around the resonant wavelength of $747 \mathrm{~nm}$. Similarly, Alrasheed and Fabrizio [15] also obtained a Rabi splitting of $76 \mathrm{meV}$ in the visible. Additionally, the strong coupling between plasmonic gap modes and photonic lattice modes was also reported by Lin et al. [23] and Saito et al. [24], respectively, and a Rabi splitting of $\sim 110 \mathrm{meV}$ was obtained [23]. Chen et al. [25] investigated the strong coupling between magnetic plasmons and photonic F-P cavity modes and obtained a Rabi-type splitting of $78 \mathrm{meV}$ around the resonant wavelength of $772 \mathrm{~nm}$.

Recently, lattice plasmons, which are usually referred to as plasmonic surface lattice resonances (SLRs), have emerged as an excellent platform for light-matter interactions on the nanoscale because of the attractive merits such as strong field enhancement extended over large volumes, and large wavelength tunability [26-28]. Taking advantage of the strong and delocalized electromagnetic fields associated with the SLRs, great progress has been achieved in the strong coupling between SLRs and different materials [10] such as organic molecules [29,30], transition metal dichalcogenides [31], and quantum dots [32]. However, the strong coupling between SLRs and photonic F-P cavity modes has not been explored yet.

In this work, we report, for the first time, the strong coupling between SLRs and photonic F-P microcavity modes by placing a thin gold film on top of a two-dimensional (2D) array of metal-insulator-metal (MIM) nanopillars. The nanopillars stand on a silica $\left(\mathrm{SiO}_{2}\right)$ substrate and are surrounded by air. As we have demonstrated previously, the SLR supported by the nanopillar array prefers such an asymmetric dielectric environment [33]. Here we will show that, the SLR can strongly couple with the photonic modes of both odd and even orders supported by the F-P microcavity formed by the thin gold film and the MIM multilayers, and splitting energies as high as $153 \mathrm{meV}$ can be obtained in the visible, corresponding to $9.5 \%$ of the resonant energy. Remarkably, we will also show that the high-energy upper polariton inherits the characteristics of the SLR but has doubled quality factor compared with the SLR.

\section{Structure Design and Numerical Setups}

Figure 1a illustrates the strongly coupled plasmonic-photonic system under study, which is composed of a 2D periodic array of MIM nanopillars standing on a silica substrate and a thin gold film of thickness $20 \mathrm{~nm}$ on the top. Here $20 \mathrm{~nm}$ is adopted for the gold film in order to compromise the transmittance through the film and the reflectance by the film $[6,11,15,19,20,25]$. The distance between the bottom surface of the gold film and the top surface of the MIM nanopillar is $h$. The nanopillars with square-shaped cross section have side length of $200 \mathrm{~nm}$ and period of $\Lambda=510 \mathrm{~nm}$ in both the $x$ and $y$ directions. The thicknesses of both the top and bottom gold nanoridges are $140 \mathrm{~nm}$, and the thickness of the central insulating silica layer is $260 \mathrm{~nm}$. The geometrical parameters of the MIM nanopillars are carefully optimized in order to maximize the splitting energy with a starting point taken from our previous work [33].

The structure is normally illuminated by a plane wave with electric field of $x$ polarization and of unitary amplitude. The reflectance spectra, as well as the near-field distributions of the plasmonic-photonic coupling system were simulated using an in-house package for fully vectorial rigorous coupled-wave analysis (RCWA) developed following [34-36]. RCWA, also known as Fourier modal method, is a powerful algorithm and is one of the most popular methods for the modeling of periodic optical structures such as gratings, metamaterials and metasurfaces. As references, we also calculated the optical properties of 
the reference F-P cavity formed by the top gold film and the MIM multilayers on the silica substrate, and the 2D array of MIM nanopillars, which are illustrated by Figure 1b,c, respectively. In all the simulations, we adopted retained orders of $41 \times 41$, which were shown to be large enough to reach the convergence regime. Wavelength-dependent permittivities of gold are taken from Johnson and Christy [37], and the refractive indices of the substrate and the central silica are set to be $n_{\text {sub }}=n_{\mathrm{I}}=1.45$.

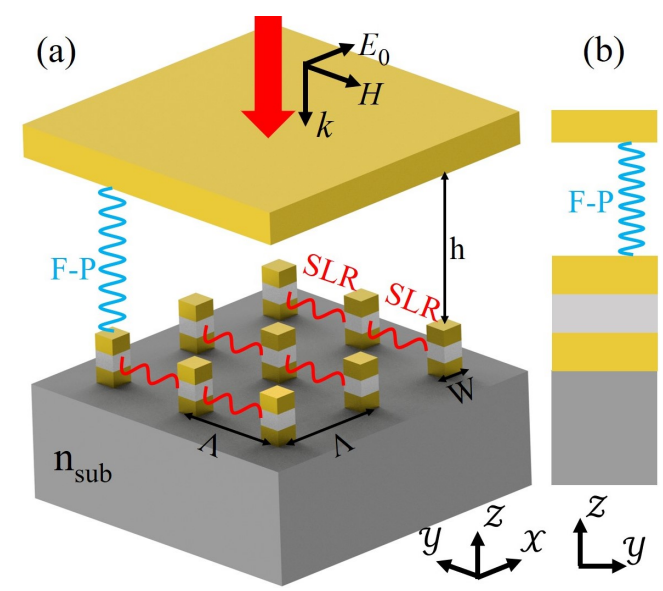

(c)

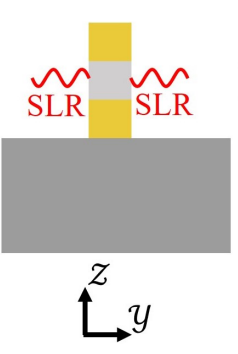

Figure 1. (a) Schematic of the strong coupling between the SLR and the F-P resonance in the proposed structure composed of a thin metal film and a 2D array of MIM nanopillars on a quartz substrate. $(\mathbf{b}, \mathbf{c})$ schematics of the side views of $(\mathbf{b})$ the reference F-P cavity that supports photonic resonances and (c) the MIM nanopillar array that supports SLR. (d) Simulated reflectance spectra of the proposed plasmonic-photonic system (red curve), the reference F-P cavity (black curve) and the MIM nanopillar array (blue curve). "UP" and "LP" represent upper polariton and lower polariton, respectively. The calculations were performed with $h=320 \mathrm{~nm}$.

\section{Results and Discussion}

Figure $1 \mathrm{~d}$ depicts the simulated reflectance spectra of the proposed plasmonic-photonic coupling system and the reference F-P cavity with $h=320 \mathrm{~nm}$, and the MIM nanopillar array. Results show that for the MIM nanopillar array in asymmetric dielectric environment, the reflectance spectra exhibits Fano-type asymmetric peak-and-dip spectral feature. A narrow dip is centered at $\lambda_{\mathrm{SLR}}=766.8 \mathrm{~nm}$ (i.e., $1.617 \mathrm{eV}$ ) and has full-width-half-maximum of $20.6 \mathrm{~nm}$. Thus the quality factor is estimated to be $Q=37$, which is about twice of that of typical localized plasmons ( $Q \sim 20$ in the visible). According to our previous study [33], this dip should correspond to a SLR, which will be further confirmed later. For the reference F-P cavity, photonic resonance can be observed at $\lambda_{\mathrm{F}-\mathrm{P}}=769.3 \mathrm{~nm}$ with the linewidth of $34.9 \mathrm{~nm}$ and the quality factor of $Q=22$. For the plasmonic-photonic coupling system, we observe that the SLR (blue curve) is split into two modes (red curve) that are resonant at $\lambda_{\mathrm{UP}}=744.1 \mathrm{~nm}$ (i.e., $1.666 \mathrm{eV}$ ) and $\lambda_{\mathrm{LP}}=819.6 \mathrm{~nm}$ (i.e., $1.513 \mathrm{eV}$ ). Strikingly, the high-energy upper polariton (UP) has much narrower linewidth of $11.6 \mathrm{~nm}$ compared with the SLR in the MIM nanopillar array and the reference F-P resonance. This corresponds to higher quality factor of $Q=64$, which is almost twice of that of the SLR and three times of that of the reference F-P resonance. In contrast, the low-energy lower polariton (LP) has wider linewidth of $31.4 \mathrm{~nm}$, corresponding to a relatively small quality factor of $Q=26$, which is comparable to that of the reference F-P cavity. In other words, thanks to the strong coupling effect, the quality factor is significantly improved for high-energy upper polariton.

The Rabi-analogue splitting energy reaches $153 \mathrm{meV}(=1.666 \mathrm{eV}-1.513 \mathrm{eV})$, which is about $9.5 \%$ of the resonance energy $(1.617 \mathrm{eV})$ and thus meets a criterion of strong couplings [6]. Moreover, the ratios of the splitting $(75.5 \mathrm{~nm})$ and the linewidths for the two polaritons (11.6 $\mathrm{nm}$ and $31.4 \mathrm{~nm}$ ) are 6.5 and 2.4, respectively, both of which are larger than unity and thus meet another criterion of strong couplings [6]. These results confirm that the proposed plasmonic-photonic system is in the strong coupling regime. Although 
this splitting energy of $153 \mathrm{meV}$ obtained in the visible regime is smaller than those in the infrared regime [11,19], it is much larger than those of many plasmonic-photonic strong coupling systems operating in the visible [15,21-23,25,38].

In order to gain a deeper insight concerning the nature of the upper and lower polaritons, in Figure 2 we plot the near-field electric field distributions $\left(|E|^{2}\right)$ at these four resonance wavelengths. Figure 2 a shows that at $\lambda_{\mathrm{SLR}}=766.8 \mathrm{~nm}$, in-plane dipolar oscillations and out-of-plane quadrupolar oscillations are excited in the top and bottom gold ridges, respectively. The electric field is greatly enhanced around the gold corners while extending over large volumes. These near-field characteristics are consistent with our previous study [33], further validating the excitation of SLR. Figure $2 b$ shows the electric field distributions of the reference F-P cavity at $\lambda_{\mathrm{F}-\mathrm{P}}=769.3 \mathrm{~nm}$. It is evident that the first-order F-P resonance is excited.

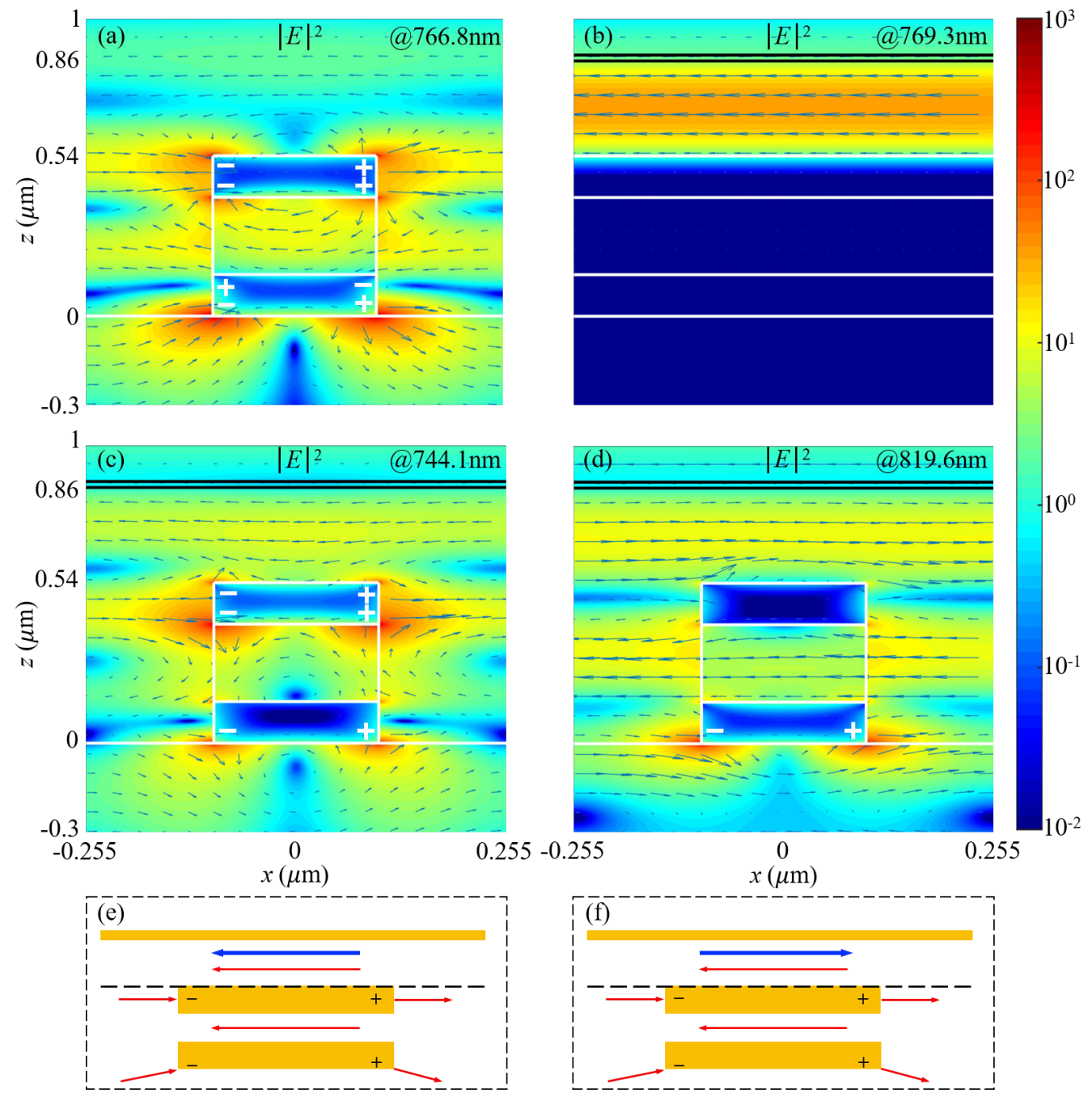

Figure 2. Simulated electric field distributions $|E|^{2}$ of (a) the MIM nanopillar array at $\lambda_{\mathrm{SLR}}=766.8 \mathrm{~nm}$, (b) the reference F-P cavity at $\lambda_{\mathrm{F}-\mathrm{P}}=769.3 \mathrm{~nm},(\mathbf{c}, \mathbf{d})$ the proposed structure of (c) $\lambda_{\mathrm{UP}}=744.1 \mathrm{~nm}$ and $\lambda_{\mathrm{LP}}=819.6 \mathrm{~nm}$. The MIM nanopillar array in $(\mathbf{a}, \mathbf{c}, \mathbf{d})$ or the MIM multilayers in $(\mathbf{b})$ are outlined by white lines and the thin metal film on the top is outlined by black lines. "+" and "-" in (a,c,d) indicate charge distributions. $(\mathbf{e}, \mathbf{f})$ Schematics of the electric field hybridization between the first-order F-P resonance (indicated by blue line) and the SLR (indicated by red lines).

For the proposed plasmonic-photonic coupling system, Figure 2c,d show the electric field distribution maps for the upper and lower polaritons locating at $\lambda_{\mathrm{UP}}=744.1 \mathrm{~nm}$ and $\lambda_{\mathrm{LP}}=819.6 \mathrm{~nm}$. Results show that for the high-energy upper polariton at $\lambda_{\mathrm{UP}}=744.1 \mathrm{~nm}$, the electric field on the top gold ridge is enhanced with large volume extension. This 
is probably because the electric field above the top gold ridge for the SLR has the same direction as that for the reference F-P cavity mode, as illustrated by Figure 2e. This nearfield feature resembles that of SLRs. For the low-energy lower polariton at $\lambda_{\mathrm{LP}}=819.6 \mathrm{~nm}$, however, because the electric fields around the top gold ridge are in the opposite direction for the SLR and for the reference F-P cavity mode, as illustrated by Figure $2 \mathrm{f}$, the fields around the top gold ridge corners are suppressed. Therefore, the resulting electric field distributions resemble those of the second-order F-P cavity mode. In other words, the high-energy upper polariton inherits more from the SLR, whereas the low-energy lower polariton inherits more from the photonic F-P resonance. Concomitantly, as we have pointed out previously, the former has much larger quality factor of $Q=64$ than the SLR, whereas the latter has relatively small quality factor of $Q=26$, which is comparable to the F-P modes.

We now investigate the effects of the F-P cavity thickness on the plasmonic-photonic strong coupling system. Figure 3 a depicts the photonic resonant modes of the reference F-P cavity with thicknesses varying from $h=0.1 \mu \mathrm{m}$ to $h=2.5 \mu \mathrm{m}$. Figure $3 \mathrm{~b}$ shows that the interactions between the photonic F-P resonant modes and the SLR result in anticrossings of these modes. Remarkably, all orders of photonic F-P resonances can interact with the SLR supported by the MIM nanopillar array. This behavior is distinct from that of the strong coupling between localized plasmons and photonic F-P modes, for which the former can interact with every second photonic F-P modes (odd or even, depending on the characteristics of the localized plasmons) $[6,11,19]$. As the F-P resonance order increases, the splitting energy decreases significantly. This is similar to the strong coupling between localized plasmons and photonic F-P modes.
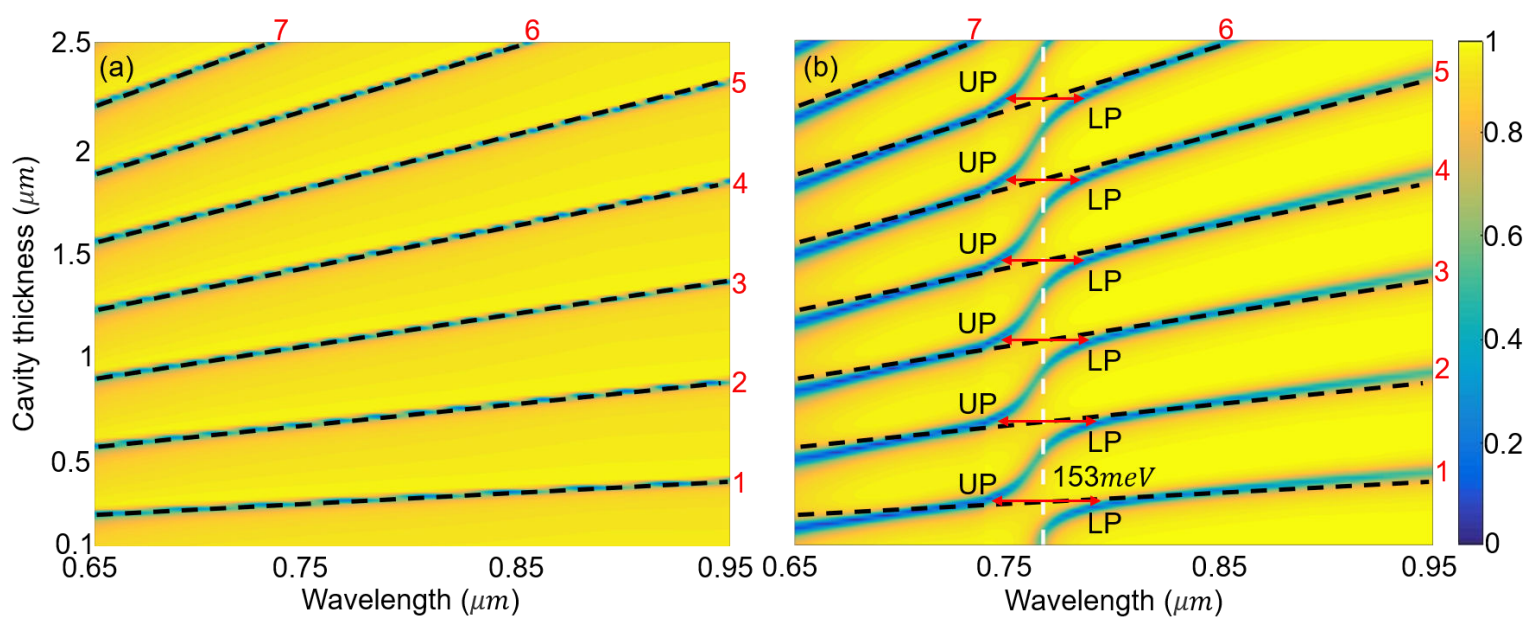

Figure 3. Simulated reflectance spectra of (a) reference F-P cavity and (b) proposed plasmonicphotonic coupling system as functions of the cavity length. The vertical white dashed line in (b) indicates the SLR wavelength of the MIM nanopillar array. Anti-crossings indicated by red arrows are observable around every intersection of the F-P modes and the SLR mode.

Figure 4 depict the near-field electric fields due to the hybridization of the SLR and the reference F-P resonances of the second and third orders. It is clear that the electric field distributions of the high-energy upper polariton exhibit strong enhancement around the gold top corners with large volume extension, resembling the feature of the SLR. For the low-energy lower polariton, however, the electric fields around the top gold corners are suppressed, resulting in similar distributions of the photonic F-P resonances. These behaviors are similar to those in Figure 2c,d. In other words, the upper polariton inherits characteristics more from the SLR, whereas the lower polariton inherits more from the photonic F-P resonances. 

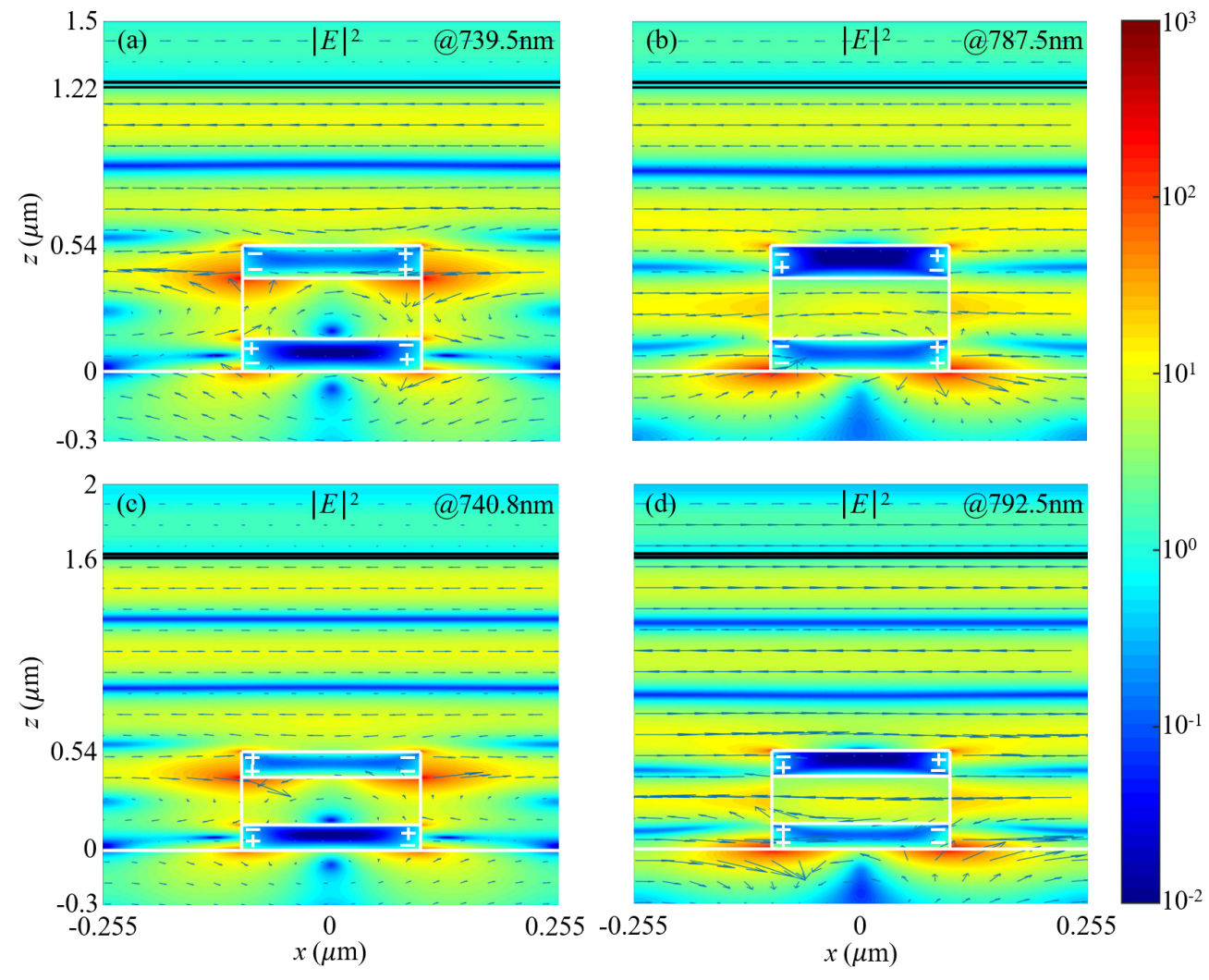

Figure 4. Similar to Figure 2c,d but at (a) $\lambda_{\mathrm{UP}}=739.5 \mathrm{~nm}$ and (b) $\lambda_{\mathrm{LP}}=787.5 \mathrm{~nm}$ for $h=1.22 \mu \mathrm{m}$ supporting the third-order photonic F-P resonance, and (c) $\lambda_{\mathrm{UP}}=740.8 \mathrm{~nm}$ and (d) $\lambda_{\mathrm{LP}}=792.5 \mathrm{~nm}$ for $h=1.6 \mu \mathrm{m}$, with which the reference F-P cavity supports the fourth-order resonance.

Additionally, we also investigate the effect of the lattice period of the MIM nanopillar array. Figure 5a shows that, as the lattice period increases, the SLR wavelength increases accordingly, as indicated by the white dashed curve. For the proposed plasmonic-photonic coupling system, Figure $5 \mathrm{~b}$ shows an anti-crossing around the intersection of the reference F-P mode and the SLR. This also leads to an upper and a lower polaritons with a splitting energy of $153 \mathrm{meV}$.
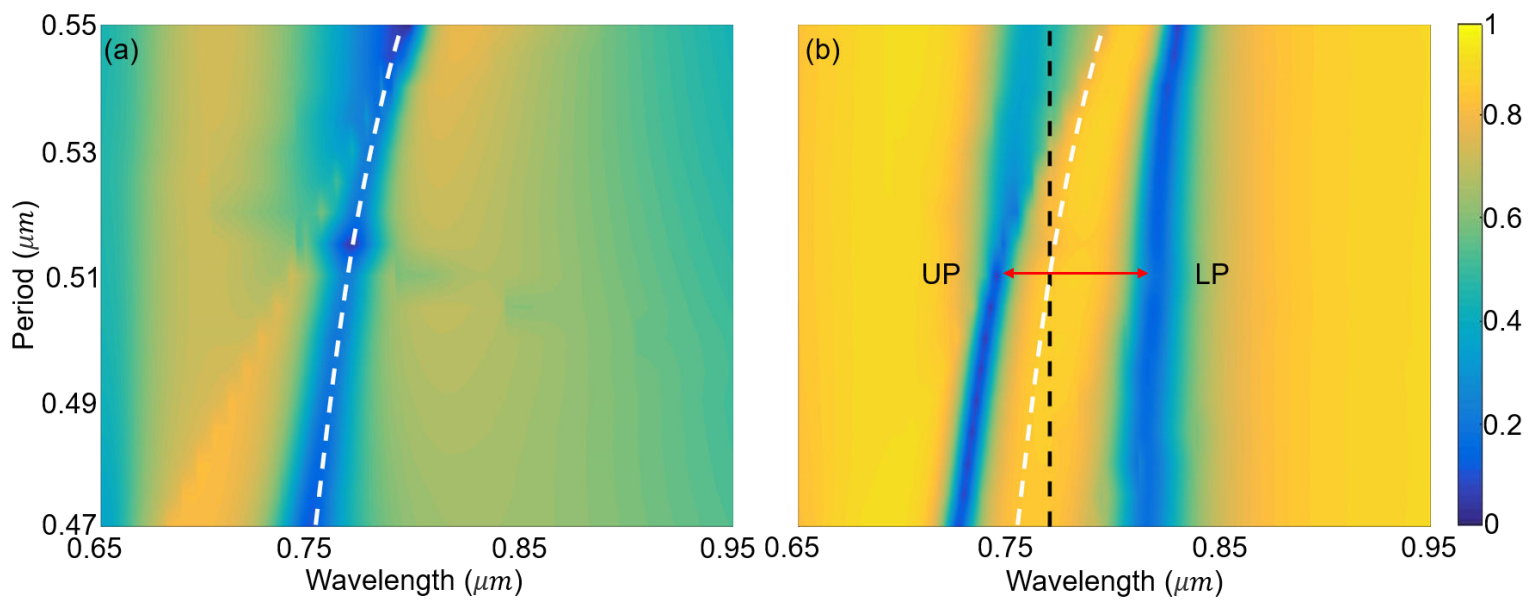

Figure 5. Simulated reflectance spectra of (a) the MIM nanopillar array and of (b) the proposed plasmonic-photonic coupling system as functions of the period. White dashed curves in $(\mathbf{a}, \mathbf{b})$ indicate the SLR, and the vertical black dashed line in $(\mathbf{b})$ indicates the wavelength of the first-order resonance of the reference F-P cavity. An anti-crossing indicated by the red arrow in (b) is observable around the intersection of the first-order reference F-P mode and the SLR. 


\section{Conclusions}

In conclusion, we have numerically observed strong coupling between plasmonic SLR and photonic F-P resonances of both even and odd orders in the proposed plasmonicphotonic coupling system. We have found that the Rabi splitting energy reaches as large as $153 \mathrm{meV}$, which is about $9.5 \%$ of the resonance energy in the visible regime. Taking advantage of the strong coupling effect, the obtained upper polariton has much larger quality factor than the SLR or the reference F-P resonance. For the strong coupling between the SLR and photonic F-P resonances of different order, we have found that the electric fields are greatly enhanced with large volume extension around the top metal ridge for the high-energy upper polariton, featuring the SLR, and that the electric fields look like the photonic F-P resonances for the low-energy lower polariton. Although in this work we adopted low- $Q$ photonic F-P microcavities formed by metal films, we expect similar or even better results can be obtained if one adopts extremely high- $Q$ all-dielectric microcavities such as photonic crystal cavities [39,40]. Therefore, we expect that the proposed plasmonicphotonic strong coupling system will provide a new scheme for strong coupling between plasmonic and photonic modes and a new approach for improving the quality factor of the SLR, and find potential applications in manipulation of the near field distributions and sensing. As a final remark, we mention that the difficulties associated with the fabrication of the thick MIM nanopillar array can be greatly alleviated by rotating the vertical MIM nanopillar by 90 degree, resulting in a horizontal MIM nanopillar array [41].

Author Contributions: Conceptualization, G.L.; methodology, Y.S.; software, G.L.; validation, Y.S.; formal analysis, Y.S., W.L., S.L. and G.L.; investigation, Y.S.; resources, Y.D. and G.L.; data curation, Y.S. and W.L.; writing-original draft preparation, Y.S.; writing-review and editing, T.Y., D.S., Y.D. and G.L.; visualization, Y.S.; supervision, D.S., Y.D. and G.L.; project administration, G.L.; funding acquisition, Y.D. and G.L. All authors have read and agreed to the published version of the manuscript.

Funding: This research was funded by the State Key Laboratory of Advanced Optical Communication Systems Networks, China (2020GZKF004) and Shenzhen Research Foundation (JCYJ20170413152328742).

Institutional Review Board Statement: Not applicable.

Informed Consent Statement: Not applicable.

Data Availability Statement: The data presented in this study are available on request from the corresponding author.

Conflicts of Interest: The authors declare no conflict of interest.

\section{References}

1. Najer, D.; Söllner, I.; Sekatski, P.; Dolique, V.; Löbl, M.C.; Riedel, D.; Schott, R.; Starosielec, S.; Valentin, S.R.; Wieck, A.D.; et al. A gated quantum dot strongly coupled to an optical microcavity. Sci. Adv. 2019, 575, 622-627. [CrossRef] [PubMed]

2. Satapathy, S.; Khatoniar, M.; Parappuram, D.K.; Liu, B.; John, G.; Feist, J.; Garcia-Vidal, F.J.; Menon, V.M. Selective isomer emission via funneling of exciton polaritons. Sci. Adv. 2021, 7, eabj0997. [CrossRef] [PubMed]

3. Hu, F.; Fei, Z. Recent Progress on Exciton Polaritons in Layered Transition-Metal Dichalcogenides. Adv. Opt. Mater. 2019, 8, 1901003. [CrossRef]

4. Hertzog, M.; Wang, M.; Mony, J.; Börjesson, K. Strong light-matter interactions: A new direction within chemistry. Chem. Soc. Rev. 2019, 48, 937-961. [CrossRef]

5. Kottilil, D.; Babusenan, A.; Vijayan, C.; Ji, W. Strong light-matter interaction in organic microcavity polaritons: Essential criteria, design principles and typical configurations. Eur. Phys. J. Spec. Top. 2021. [CrossRef]

6. Ameling, R.; Giessen, H. Microcavity plasmonics: Strong coupling of photonic cavities and plasmons. Laser Photonics Rev. 2013, 7, 141-169. [CrossRef]

7. Baranov, D.G.; Wersall, M.; Cuadra, J.; Antosiewicz, T.J.; Shegai, T. Novel Nanostructures and Materials for Strong Light-Matter Interactions. ACS Photonics 2018, 5, 24-42. [CrossRef]

8. Dovzhenko, D.S.; Ryabchuk, S.V.; Rakovich, Y.P.; Nabiev, I.R. Light-matter interaction in the strong coupling regime: Configurations, conditions, and applications. Nanoscale 2018, 10, 3589-3605. [CrossRef]

9. Yu, X.; Yuan, Y.; Xu, J.; Yong, K.T.; Qu, J.; Song, J. Strong Coupling in Microcavity Structures: Principle, Design, and Practical Application. Laser Photonics Rev. 2019, 13, 1800219. [CrossRef]

10. Ramezani, M.; Berghuis, M.; Rivas, J.G. Strong light-matter coupling and exciton-polariton condensation in lattices of plasmonic nanoparticles [Invited]. J. Opt. Soc. Am. B 2019, 36, E88-E103. [CrossRef] 
11. Ameling, R.; Giessen, H. Cavity Plasmonics: Large Normal Mode Splitting of Electric and Magnetic Particle Plasmons Induced by a Photonic Microcavity. Nano Lett. 2010, 10, 4394-4398. [CrossRef] [PubMed]

12. Ameling, R.; Langguth, L.; Hentschel, M.; Mesch, M.; Braun, P.V.; Giessen, H. Cavity-enhanced localized plasmon resonance sensing. Appl. Phys. Lett. 2010, 97, 253116. [CrossRef]

13. Schmidt, M.A.; Lei, D.Y.; Wondraczek, L.; Nazabal, V.; Maier, S.A. Hybrid nanoparticle-microcavity-based plasmonic nanosensors with improved detection resolution and extended remote-sensing ability. Nat. Commun. 2012, 3, 1108. [CrossRef] [PubMed]

14. Chen, J.; Nie, H.; Peng, C.; Qi, S.; Tang, C.; Zhang, Y.; Wang, L.; Park, G.S. Enhancing the Magnetic Plasmon Resonance of Three-Dimensional Optical Metamaterials via Strong Coupling for High-Sensitivity Sensing. J. Light. Technol. 2018, 36, $3481-3485$. [CrossRef]

15. Alrasheed, S.; Fabrizio, E.D. Effect of Surface Plasmon Coupling to Optical Cavity Modes on the Field Enhancement and Spectral Response of Dimer-Based sensors. Sci. Rep. 2017, 7, 10524. [CrossRef]

16. Li, B.; Zu, S.; Zhang, Z.; Zheng, L.; Jiang, Q.; Du, B.; Luo, Y.; Gong, Y.; Zhang, Y.; Lin, F.; et al. Large Rabi splitting obtained in Ag-WS2 strong-coupling heterostructure with optical microcavity at room temperature. Opto-Electron. Adv. 2019, 2, 190008. [CrossRef]

17. Bisht, A.; Cuadra, J.; Wersall, M.; Canales, A.; Antosiewicz, T.J.; Shegai, T. Collective Strong Light-Matter Coupling in Hierarchical Microcavity-Plasmon-Exciton Systems. Nano Lett. 2019, 19, 189-196. [CrossRef]

18. Li, P.; Ji, L.; Gao, N.; Wang, H.; Ge, S.; Huang, K.; Kang, J.; Yu, E.T. Peak splitting and locking behavior arising from Fano interference between localized surface plasmons and cavity modes. Phys. Rev. B 2019, 99, 125420. [CrossRef]

19. Baranov, D.G.; Munkhbat, B.; Zhukova, E.; Bisht, A.; Canales, A.; Rousseaux, B.; Johansson, G.; Antosiewicz, T.J.; Shegai, T. Ultrastrong coupling between nanoparticle plasmons and cavity photons at ambient conditions. Nat. Commun. 2020, 11, 2715. [CrossRef]

20. Ameling, R.; Dregely, D.; Giessen, H. Strong coupling of localized and surface plasmons to microcavity modes. Opt. Lett. 2011, 36, 2218-2220. [CrossRef]

21. Chen, S.; Li, G.; Lei, D.; Cheah, K.W. Efficient energy exchange between plasmon and cavity modes via Rabi-analogue splitting in a hybrid plasmonic nanocavity. Nanoscale 2013, 5, 9129-9133. [CrossRef]

22. Wang, Y.; Sun, C.; Gan, F.; Li, H.; Gong, Q.; Chen, J. Sharp phase variations from the plasmon mode causing the Rabi-analogue splitting. Nanophotonics 2017, 6, 1101-1107. [CrossRef]

23. Lin, Q.Y.; Li, Z.; Brown, K.A.; O’Brien, M.N.; Ross, M.B.; Zhou, Y.; Butun, S.; Chen, P.C.; Schatz, G.C.; Dravid, V.P.; et al. Strong Coupling between Plasmonic Gap Modes and Photonic Lattice Modes in DNA-Assembled Gold Nanocube Arrays. Nano Lett. 2015, 15, 4699-4703. [CrossRef]

24. Saito, H.; Yoshimoto, D.; Lourenco-Martins, H.; Yamamoto, N.; Sannomiya, T. Hybridization of Gap Modes and Lattice Modes in a Plasmonic Resonator Array with a Metal-Insulator-Metal Structure. ACS Photonics 2019, 6, 2618-2625. [CrossRef]

25. Chen, J.; Zhang, T.; Tang, C.; Mao, P.; Liu, Y.; Yu, Y.; Liu, Z. Optical Magnetic Field Enhancement via Coupling Magnetic Plasmons to Optical Cavity Modes. IEEE Photon. Technol. Lett. 2016, 28, 1529-1532. [CrossRef]

26. Wang, W.; Ramezani, M.; Väkeväinen, A.I.; Törmä, P.; Rivas, J.G.; Odom, T.W. The Rich Photonic World of Plasmonic Nanoparticle Arrays. Mater. Today 2018, 21, 303-314. [CrossRef]

27. Kravets, V.G.; Kabashin, A.V.; Barnes, W.L.; Grigorenko, A.N. Plasmonic Surface Lattice Resonances: A Review of Properties and Applications. Chem. Rev. 2018, 118, 5912-5951. [CrossRef]

28. Wang, D.; Guan, J.; Hu, J.; Bourgeois, M.R.; Odom, T.W. Manipulating Light-Matter Interactions in Plasmonic Nanoparticle Lattices. Acc. Chem. Res. 2019, 52, 2997-3007. [CrossRef]

29. Väkeväinen, A.I.; Moerland, R.J.; Rekola, H.T.; Eskelinen, A.P.; Martikainen, J.P.; Kim, D.H.; Törmä, P. Plasmonic Surface Lattice Resonances at the Strong Coupling Regime. Nano Lett. 2014, 14, 1721-1727. [CrossRef]

30. Shi, L.; Hakala, T.K.; Rekola, H.T.; Martikainen, J.P.; Moerland, R.J.; Törmä, P. Spatial Coherence Properties of Organic Molecules Coupled to Plasmonic Surface Lattice Resonances in the Weak and Strong Coupling Regimes. Phys. Rev. Lett. 2014, $112,153002$. [CrossRef]

31. Wang, S.; Le-Van, Q.; Vaianella, F.; Maes, B.; Barker, S.E.; Godiksen, R.H.; Curto, A.G.; Rivas, J.G. Limits to Strong Coupling of Excitons in Multilayer WS2 with Collective Plasmonic Resonances. ACS Photonics 2019, 6, 286-293. [CrossRef]

32. Yadav, R.K.; Bourgeois, M.R.; Cherqui, C.; Juarez, X.G.; Wang, W.; Odom, T.W.; Schatz, G.C.; Basu, J.K. Room Temperature Weak-to-Strong Coupling and the Emergence of Collective Emission from Quantum Dots Coupled to Plasmonic Arrays. ACS Nano 2020, 14, 7347-7357. [CrossRef]

33. Yang, X.; Xiao, G.; Lu, Y.; Li, G. Narrow plasmonic surface lattice resonances with preference to asymmetric dielectric environment. Opt. Express 2019, 27, 25384-25394. [CrossRef]

34. Moharam, M.G.; Pommet, D.A.; Grann, E.B.; Gaylord, T.K. Stable Implementation of the Rigorous Coupled-Wave Analysis for Surface-Relief Gratings: Enhanced Transmittance Matrix Approach. J. Opt. Soc. Am. A 1995, 12, 1077-1086. [CrossRef]

35. Lalanne, P. Improved formulation of the coupled-wave method for two-dimensional gratings. J. Opt. Soc. Am. A 1997, 14, 1592-1598. [CrossRef]

36. David, A.; Benisty, H. Fast factorization rule and plane-wave expansion method for two-dimensional photonic crystals with arbitrary hole-shape. Phys. Rev. B 2006, 73, 075107. [CrossRef]

37. Johnson, P.B.; Christy, R.W. Optical Constants of the Noble Metals. Phys. Rev. B 1972, 6, 4370-4379. [CrossRef]

38. Alast, F.H.; Li, G.; Cheah, K.W. Rabi-like splitting from large area plasmonic microcavity. AIP Adv. 2017, 7, 085201. [CrossRef] 
39. Kassa-Baghdouche, L.; Boumaza, T.; Cassan, E.; Bouchemat, M. Enhancement of $Q$-factor in SiN-based planar photonic crystal L3 nanocavity for integrated photonics in the visible-wavelength range. Optik 2015, 126, 3467-3471. [CrossRef]

40. Kassa-Baghdouche, L.; Boumaza, T.; Bouchemat, M. Optimization of $Q$-factor in nonlinear planar photonic crystal nanocavity incorporating hybrid silicon/polymer material. Phys. Scr. 2015, 90, 065504. [CrossRef]

41. Fang, X.; Xiong, L.; Shi, J.; Li, G. High-Q quadrupolar plasmonic lattice resonances in horizontal metal-insulator-metal gratings. Opt. Lett. 2021, 46, 1546-1549. [CrossRef] [PubMed] 